\title{
Focus sur le traitement comptable des brevets : les règles d'évaluation et de comptabilisation des brevets aboutissent-elles à de l'information utile aux
} investisseurs?

\section{Focus on the accounting treatment of patents: Do patent assessment and recognition rules lead to useful information for investors?}

\author{
Lionel Touchais $^{1}$ \\ ${ }^{1}$ Université Paris Nanterre, CEROS, lionel.touchais@parisnanterre.fr
}

\begin{abstract}
RÉSUMÉ. Les investissements immatériels réalisés par les entreprises ne sont pas toujours correctement intégrés en comptabilité. S'agissant des brevets, selon le référentiel comptable et le mode d'acquisition (achat ou fabrication en interne), les impacts sur le bilan (la valeur comptable du brevet) et sur le compte de résultat (le résultat de l'exercice) peuvent être fortement différents. La problématique de l'évaluation et de la reconnaissance comptable des éléments incorporels constitue un axe de recherche majeur dans le domaine de la comptabilité. De nombreux travaux se sont interrogés sur leur contenu informationnel et aboutissent à deux résultats majeurs. D'une part, une activation des frais de développement (inscription d'un actif incorporel au bilan) apporte de l'information utile aux investisseurs en faisant ressortir les projets susceptibles de dégager des flux de trésorerie futurs. D'autre part, diffuser de l'information volontaire sur les résultats de l'activité de recherche et développement (R\&D) au travers de données relatives aux brevets permet de réduire l'asymétrie d'information liée aux intangibles.

ABSTRACT. The intangible investments made by companies are not always correctly recognized in financial statements. For patents, according to the accounting standards used and the way the firms obtain them (purchased or internally produced), the financial consequences on the balance sheet (the book value of the patent) and the income statement (the profit and loss) are profoundly different. The question about the assessment and the recognition of intangible items is a major research stream in the field of accounting. Many scholars work on the informational content of the intangibles recorded in financial statements. They demonstrate two main outcomes. On the one hand, with a capitalization of development costs (recognition of an intangible asset in the balance sheet), firms give useful information to investors about the potential future cash flows of their development projects. On the other hand, the information asymmetry related to intangibles can be reduced by disclosing voluntary information about the research and development activity with measures of innovation outputs related to patents.

MOTS-CLÉS. Brevets, Incorporels, Evaluation, Comptabilisation, Impacts financiers.

KEYWORDS. Patents. Intangibles, Assessment, Recognition, Financial impacts.
\end{abstract}

La comptabilité a été développée à une époque où les incorporels étaient insignifiants. Elle ne permet pas d'intégrer de manière satisfaisante les éléments intangibles (notoriété, savoir-faire, capacité d'innovation, qualité du management...) qui constituent aujourd'hui la principale source de croissance et de création de valeur des firmes [LEV 03]. Certains immatériels sont inscrits au bilan comme actifs incorporels, d'autres sont considérés comme des coûts (et non des investissements) alors même qu'ils participent également au développement du capital immatériel de l'entreprise. Des auteurs expliquent d'ailleurs la dégradation du caractère prédictif et informationnel des données comptables par le développement des immatériels qui sont mal appréhendés en comptabilité [LEV 99].

La comptabilisation des brevets relève de la problématique des immobilisations incorporelles qui constitue un domaine de recherche majeur en comptabilité. Selon le référentiel comptable utilisé, en raison de positionnements différents, les règles d'évaluation et de reconnaissance des immatériels diffèrent avec, pour conséquence, des valeurs comptables des brevets au bilan susceptibles d'être 
fortement différentes selon le lieu d'implantation de l'entreprise. Le traitement de ces incorporels diffère également fortement en fonction du mode d'acquisition (achat versus fabrication en interne).

Dans le cadre de ce numéro spécial traitant des brevets, il nous semble important d'analyser les règles d'évaluation et de reconnaissance comptable des brevets afin de mettre en exergue leurs conséquences sur la valorisation de ces actifs au bilan des entreprises et leur impact sur le résultat de l'exercice publié. Cela nous conduit ensuite à nous interroger sur leur contenu informationnel dans les états financiers.

Après avoir présenté les principaux traitements comptables existant pour les brevets dans trois référentiels comptables (les règles françaises, les normes internationales : les IAS et les IFRS ${ }^{1}$, les US GAAP ${ }^{2}$ ) (partie 1), nous nous interrogeons sur le contenu informationnel de ces données financières (partie 2).

\section{Les brevets dans les états financiers : quelles règles comptables ?}

L'intégration des brevets dans les états financiers est différente selon qu'il s'agit de la phase initiale d'achat ou de production (paragraphe 1.1.) ou de la phase d'utilisation du brevet (paragraphe 1.2.). Pour faciliter la compréhension, nous illustrons nos développements avec le cas du groupe Sanofi en nous appuyant sur son rapport annuel 2018 élaboré avec le référentiel comptable international : les IAS et IFRS.

\subsection{La phase initiale}

Selon le mode d'acquisition : achat ou production en interne, la prise en compte comptable des brevets diffère très fortement. Un brevet acheté est inscrit au bilan de l'acheteur en immobilisations incorporelles pour son coût d'achat. Le coût d'achat comprend les principaux éléments suivants : le prix d'achat et les frais directement liés à l'acquisition du brevet et à sa préparation avec, par exemple, les honoraires et les commissions liés à l'acquisition. Par exception à la règle, les frais de formation visant à préparer les salariés à utiliser le brevet ne peuvent pas être activés. Ils doivent être inscrits en charges, d'où un impact négatif immédiat sur le résultat de l'exercice et une valeur comptable du brevet minorée.

Dans le cas d'un brevet produit en interne par l'entreprise, la situation est très différente. Avant de déposer un brevet, les dépenses relatives au développement de l'innovation sont considérées comme des frais de recherche et développement dont le traitement comptable diffère selon le référentiel utilisé. Avec les règles comptables internationales, les IAS et les IFRS, il convient de distinguer les phases de recherche et de développement.

-Le projet relève de la phase de recherche lorsque l'entreprise travaille à la production de nouvelles connaissances. Il s'agit d'une « investigation originale et programmée entreprise en vue d'acquérir une compréhension et des connaissances scientifiques ou techniques nouvelles » [IAS 04]. Au regard de l'incertitude portant sur l'aboutissement du projet et sur sa transformation en un

\footnotetext{
${ }^{1}$ IAS (International Accounting Standards) et IFRS (International Financial Reporting Standards) sont les deux acronymes désignant le référentiel comptable international qui est obligatoire pour les états financiers consolidés des groupes cotés sur les marchés règlementés de l'Union Européenne. Ces normes comptables sont utilisées dans plus de 130 pays dans le monde, essentiellement pour les états financiers des groupes cotés. Les règles d'évaluation, de comptabilisation et d'information traitant des immobilisations incorporelles relèvent de I'IAS 38. Pour plus d'informations, se reporter à Touchais L., " Comptabilité internationale : les IFRS », Économica, 2ème édition, 2019.

${ }^{2}$ Les US GAAP (United States Generally Accepted Accounting Principles) constituent le référentiel comptable applicable aux Etats-Unis.
} 
actif générant des avantages économiques futurs (flux nets de trésorerie futurs), toutes les dépenses relatives à la phase de recherche sont considérées comme des charges à inscrire au compte de résultat. Alors même qu'elles constituent un investissement susceptible de contribuer à la création d'un actif incorporel et donc être sources de valeur future, elles ne peuvent pas être inscrites au bilan comme une immobilisation. Elles sont, au contraire, considérées comme un coût avec un impact négatif immédiat sur le résultat de l'entreprise.

- La phase de développement consiste à utiliser ses connaissances pour développer ou améliorer un produit ou un procédé. Il s'agit d'appliquer les « résultats de la recherche ou d'autres connaissances à un plan ou un modèle en vue de la production de matériaux, dispositifs, produits, procédés, systèmes ou services nouveaux ou substantiellement améliorés avant le commencement de leur production commerciale ou de leur utilisation » [IAS 04]. Lorsque l'entreprise est en mesure de démontrer que le projet présente une forte probabilité de réussite, les dépenses liées à sa production doivent être activées. En d'autres termes, elles sont inscrites au bilan comme éléments constitutifs de la fabrication de l'immatériel susceptible de faire ensuite l'objet d'un brevet. Cette forte probabilité de réussite suppose le respect d'un certain nombre de conditions. Pour un projet de développement donné, l'entreprise doit ainsi démontrer qu'elle a l'intention et la capacité (faisabilité technique et disponibilité des ressources nécessaires) de terminer l'actif (l'innovation), de le vendre ou de l'utiliser (existence d'un marché en cas de vente ou utilité de l'immobilisation en cas d'utilisation en interne) de manière à en obtenir des avantages économiques (flux nets de trésorerie) futurs probables. Il convient également d'être en mesure d'évaluer de manière relativement fiable les dépenses relatives à ce projet. Sans système d'information permettant d'avoir une certaine traçabilité des frais liés aux différents projets de développement, il n'est pas possible de distinguer les coûts relatifs aux projets nécessitant une activation des autres. Les frais inscrits au bilan comme éléments constitutifs de l'immobilisation incorporelle comprennent toutes les dépenses directement liées à la création, la production et la préparation de l'actif en vue de son fonctionnement, à savoir : la consommation de matières premières et de prestations de services liée au projet, le coût du personnel impliqué dans la production de l'actif, les coûts de dépôt de brevet... et les intérêts d'emprunt supportés pendant la période de développement (si elle est significativement importante). Les éventuels frais commerciaux et frais généraux ne peuvent pas être activés.

En résumé, sur 1'ensemble des coûts liés à la «fabrication » du brevet, seules les dépenses de développement supportées à partir de la date à laquelle l'incertitude sur les revenus futurs peut être raisonnablement levée sont inscrites au bilan. Il n'est pas possible d'intégrer les dépenses antérieures relatives au projet. La définition du point de basculement est donc particulièrement importante. Comptablement, par prudence, c'est-à-dire pour ne pas prendre le risque d'enregistrer un actif incorporel «sans valeur », les dépenses préalables sont considérées comme un coût pour l'entreprise et non comme des éléments constitutifs d'un investissement immatériel. Ce traitement comptable se traduit par des brevets qui apparaissent dans les états financiers pour des montants largement sous-évalués lorsqu'ils sont produits en interne.

Ces règles comptables posent des difficultés car elles se traduisent par une valorisation des brevets différente selon la politique d'acquisition de l'entreprise. Alors que les brevets achetés sont inscrits en immobilisation incorporelle pour leur coût d'achat, ceux développés en interne ne reprennent que les dépenses de développement supportés à partir de la date à laquelle les conditions pour une activation sont réunies, en d'autres termes, lorsque l'incertitude sur les revenus futurs est levée. Or, en règle générale, à cette date, la plupart des frais a déjà été supportée. En d'autres termes, lorsque l'entreprise privilégie un développement en interne, les brevets sont nécessairement sousévalués dans les états financiers. Un certain nombre de dépenses liées à ces investissements sont considérés comme des charges et viennent donc réduire le résultat de l'exercice alors qu'elles constituent un investissement immatériel, source de création de valeur future, sur le plan économique. 
Les actifs incorporels sont «initialement évalués à leur coût d'acquisition ou à leur coût de production pour Sanofi, comprenant tous les coûts directement attribuables à la préparation de leur utilisation ».

Pour les travaux de R\&D réalisés en interne, « en raison des risques et incertitudes liés aux autorisations réglementaires et au processus de recherche et de développement », les conditions liées à l'activation des frais de développement « ne sont pas réputées remplies avant l'obtention de l'autorisation de mise sur le marché des médicaments (AMM) en règle générale. Par conséquent, les frais de développement internes intervenant avant l'obtention de l'AMM, principalement composés des coûts des études cliniques, sont généralement comptabilisés en charges (...) dès lorsqu'ils sont encourus ». Après l'obtention de l'AMM, les frais de développement susceptibles d'être activés sont relativement limités. En d'autres termes, les dépenses inscrites à l'actif en frais de développement au titre de brevets futurs sont très faibles au regard des dépenses supportées par le groupe pour développer un principe actif.

Pour la R\&D acquise par le groupe, « les montants versés à des tiers sous forme de paiement initial ou de paiements d'étapes relatifs à des spécialités pharmaceutiques n'ayant pas encore obtenu d'autorisation de mise sur le marché sont comptabilisés à l'actif ».

Comptablement, les brevets développés en interne sont donc très largement sous-évalués au bilan. Ce n'est pas le cas des brevets résultant d'acquisition de travaux de recherche et développement qui font l'objet d'une inscription automatique au bilan.

Les règles relatives aux frais de développement peuvent être différentes selon le référentiel comptable utilisé par les entreprises. Aux Etats-Unis, avec les US GAAP, les frais de développement relatifs à un brevet sont toujours inscrits en charges au compte de résultat de l'exercice où ils interviennent au motif qu'il n'est jamais certain qu'un tel projet aboutisse réellement à un actif, à savoir : une «innovation » susceptible de générer des flux nets de trésorerie futurs. Au nom du principe de prudence, ces investissements immatériels n'apparaissent pas au bilan mais sont considérés comme un coût alors même qu'ils contribuent à dynamiser les résultats futurs de l'entreprise. Cette situation accroît la sous-évaluation des immatériels au bilan et entraîne un décalage dans le temps entre l'inscription immédiate en charges des frais de développement des brevets et les flux de trésorerie futurs générés par le projet. Dans le cas français, sauf à utiliser les normes internationales pour les états financiers de groupes cotés sur un marché règlementé, il est fortement recommandé d'immobiliser les frais de développement des brevets à l'actif si les conditions pour une activation sont réunies. Toutefois, pour des raisons historiques, les groupes peuvent continuer à les enregistrer intégralement en charges à l'instar du traitement comptable retenue aux Etats-Unis. Selon la méthode retenue, le coût des brevets apparaîtra partiellement au bilan ou au compte de résultat.

L'inscription des frais de développement des brevets au bilan, plutôt qu'en charges, est censée apporter de l'information utile aux investisseurs sur les performances futures de l'entreprise puisque seuls les projets a priori rentables sont activés. L'inconvénient de cette reconnaissance tient toutefois à son caractère potentiellement discrétionnaire en raison de conditions à respecter relativement subjectives. Face à des difficultés momentanées, l'entreprise peut être tentée d'inscrire au bilan des dépenses de création de brevets ne respectant pas véritablement les critères dans le seul but d'améliorer de manière artificielle et provisoire son résultat et ses capitaux propres. Le risque est alors d'avoir des frais de développement de brevets au bilan sans réelle valeur car la capacité du projet à dégager des flux de trésorerie futurs n'est pas démontrée. 
Cas particuliers :

- Un brevet peut être acquis moyennant le paiement de redevances pendant une certaine durée. La valeur inscrite au bilan correspond alors à la valeur actuelle des redevances futures probables. La quote-part des redevances correspondant à la partie «intérêts » est considérée comme une charge financière impactant le résultat de l'exercice où la redevance est due.

-Avec les IFRS et les US GAAP, la notion de propriété n'est pas nécessaire pour inscrire les brevets à l'actif. Sauf exceptions, les brevets utilisés dans le cadre de contrats de location sont également considérés comme des immobilisations incorporelles. En effet, d'un point de vue économique, il n'y a pas de différences majeures entre un brevet acheté financé à l'aide d'un emprunt et un brevet loué. La location de cet incorporel permet au locataire (le preneur) de décider de la manière dont il va utiliser cet actif pour en retirer des flux de trésorerie comme s'il en était le propriétaire. Quant aux loyers, ils peuvent être assimilés à des mensualités d'emprunt. À la date de mise à disposition du brevet, il convient donc d'inscrire au bilan une immobilisation incorporelle liée au «droit d'utilisation » conféré par la location et une dette financière relative aux engagements de paiements futurs actualisés. À cette date, la valeur comptable du brevet dans les états financiers du locataire correspond, pour l'essentiel, au montant de la dette financière initiale augmentée des paiements préalables déjà réalisés et des frais directement liés à la préparation du brevet (hormis les frais de formation).

\subsection{La phase d'utilisation}

Après son inscription initiale au bilan, avec les normes comptables internationales (les IAS et les IFRS), une immobilisation incorporelle peut relever de deux traitements comptables différents avec une évaluation soit au coût (méthode du coût historique), soit à la juste valeur (méthode de la réévaluation).

Avec l'évaluation au coût, à la fin de chaque exercice comptable, le brevet apparaît au bilan pour sa valeur comptable initiale diminuée du cumul des amortissements et des dépréciations. Les amortissements consistent à reporter en charges au compte de résultat le coût du brevet sur la durée de protection légale (20 ans) ou sur la durée d'utilisation si elle est plus courte. Ils correspondent à la «consommation d'avantages économiques » de l'actif. L'amortissement commence lorsque le brevet est prêt à être utilisé : date d'achat pour un brevet acheté ou date du dépôt pour un brevet créé en interne.

Après l'amortissement, si un ou plusieurs indices (exemples: obsolescence du brevet, «performance » inférieure aux prévisions...) laissent présager une perte de valeur du brevet (une valeur comptable surévaluée par rapport à sa valeur réelle estimée : sa valeur recouvrable), un test de dépréciation doit être réalisé. Il s'agit alors de comparer la valeur comptable du brevet avec sa valeur recouvrable. La valeur recouvrable représente le montant le plus élevé de la valeur d'utilité (valeur actuelle des flux de trésorerie futurs attendus de l'utilisation de l'actif et de sa revente) et de sa juste valeur nette (valeur de marché estimée diminuée des éventuels coûts liés à sa cession). En règle générale, la valeur de marché d'un brevet n'étant pas connue, le test de perte de valeur est souvent réalisé par rapport à la valeur d'utilité qui est alors considérée comme supérieure à la juste valeur nette. L'objectif de la valeur recouvrable consiste à estimer la valeur réelle de l'actif. Lorsque ce test fait ressortir une moins-value latente (valeur comptable > valeur recouvrable), l'entreprise doit l'inscrire en charges de l'exercice et diminuer la valeur comptable du brevet pour l'aligner sur sa valeur recouvrable. Ces pertes de valeur sont réversibles. Si elles diminuent sur les années futures, elles seront réduites, voire éliminées. En revanche, en situation de sous-évaluation (valeur comptable < valeur recouvrable), la plus-value latente n'est pas prise en compte en vertu d'un principe comptable important : le principe de prudence. L'idée sous-jacente est de ne pas enregistrer les gains potentiels de crainte qu'ils ne se réalisent pas in fine. Il s'agit de ne pas prendre le risque de surévaluer l'actif et le résultat à un instant $\mathrm{t}$. 
En résumé, avec cette approche, la valeur du brevet diminue progressivement au bilan pour atteindre une valeur nulle au moment où il «tombe » dans le domaine public. Son coût aura été rapporté en charges sur toute la durée de protection (ou la durée d'utilisation si elle est plus courte).

Une autre solution consiste à utiliser la méthode de la juste valeur (fair value) qui vise à réévaluer les immobilisations incorporelles à la fin de chaque exercice en intégrant les plus ou moins-values latentes relatives au brevet. Cette méthode d'évaluation permet d'inscrire les brevets pour des montants plus proches des valeurs réelles. Cela suppose toutefois d'être en mesure d'évaluer l'immobilisation par référence à un marché actif. Cela signifie qu'il doit exister un marché avec des transactions selon une fréquence et un volume suffisants pour fournir de façon continue de l'information sur le prix. Cette situation est peu probable pour les brevets. Cette méthode de réévaluation, qui n'est d'ailleurs pas prévue avec le référentiel français, est donc peu susceptible de s'appliquer pour les brevets. Dans la pratique, cet incorporel relève donc d'une évaluation au coût historique quel que soit le référentiel comptable. En cas d'accroissement de la valeur de marché du brevet, cela signifie qu'il sera nécessairement sous-évalué au bilan de l'entreprise.

\section{Le groupe SANOFI - rapport annuel 2018 en IFRS}

Le groupe Sanofi utilise la méthode du coût historique. Ainsi, les frais de recherche et développement (qui peuvent donner lieu à des brevets) «sont comptabilisés à leur coût diminué du cumul des amortissements et, le cas échéant, du cumul des pertes de valeur ». Pour le test de perte de valeur, l'entreprise indique qu'elle s'appuie sur des «indicateurs quantitatifs et qualitatifs, dont les principaux sont des indicateurs relatifs au développement du portefeuille de recherche et développement, à la pharmacovigilance, aux litiges relatifs aux brevets et à l'arrivée de produits concurrents ».

L'évaluation au coût, présentée dans les paragraphes précédents, s'applique indifféremment aux brevets achetés ou produits en interne. Cette méthode concerne également les brevets acquis moyennant le paiement de redevances ou, avec le référentiel comptable IFRS ou US GAAP, les brevets utilisés dans le cadre d'un contrat de location qui sont également inscrits au bilan.

Pour conclure cette première section, selon le référentiel comptable utilisé (IFRS, français ou US GAAP), le positionnement du normalisateur comptable vis-à-vis des éléments de coûts relatifs aux brevets diffèrent, d'où une reconnaissance au bilan différente selon les règles utilisées. La manière dont les brevets sont obtenus (achat versus fabrication par l'entreprise) intervient également sur leur valeur comptable avec une sous-évaluation systématique lorsqu'ils sont créés en interne. Dans tous les cas, après leur inscription initiale, leur valeur au bilan ne peut pas faire l'objet d'une réévaluation visant à aligner la valeur comptable sur leur juste valeur (la valeur de marché). La valeur comptable initiale des brevets diminue avec, d'une part, des dotations aux amortissements au compte de résultat reflétant la «consommation » des avantages économiques de l'immatériel, d'autre part, des dépréciations en situation de moins-values latentes.

Le mode d'acquisition (achat ou fabrication par l'entreprise) impacte également le résultat de l'exercice publié par l'entreprise. Avec les IFRS, une fabrication en interne d'un brevet se traduit par un enregistrement immédiat des dépenses liées au projet tant que sa réussite probable n'est pas démontrée. À l'inverse, s'il est acheté, ces frais sont étalés au compte de résultat sur la durée de protection légale (ou sur la durée d'utilisation si elle est plus courte) par le biais des dotations aux amortissements. L'amortissement permet d'intégrer au résultat de l'exercice, lorsque le brevet est « opérationnel », à la fois le chiffre d'affaires généré par cet actif et son coût d'achat qui fait l'objet d'un étalement. 


\begin{tabular}{|c|c|c|c|c|}
\hline \multicolumn{2}{|c|}{ Référentiel comptable } & IFRS & $\begin{array}{l}\text { Référentiel } \\
\text { français }\end{array}$ & US GAAP \\
\hline \multirow{3}{*}{ 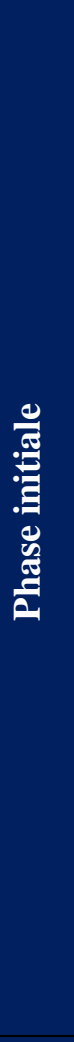 } & Achat & \multicolumn{3}{|c|}{$\begin{array}{l}\text { Inscription au bilan pour le coût d'acquisition : } \\
\text { prix d'achat et frais directs de préparation }\end{array}$} \\
\hline & Prise en location & $\begin{array}{l}\text { Inscription du «droit } \\
\text { d'utilisation à l'actif » } \\
\text { avec une « dette liée à } \\
\text { la location » }\end{array}$ & $\begin{array}{l}\text { Enregistrement des } \\
\text { loyers en charges }\end{array}$ & $\begin{array}{c}\text { Inscription du « droit } \\
\text { d'utilisation à } \\
\text { l'actif » avec une } \\
\text { « dette liée à la } \\
\text { location » }\end{array}$ \\
\hline & $\begin{array}{l}\text { Développement } \\
\text { en interne }\end{array}$ & $\begin{array}{l}\text { Activation obligatoire } \\
\text { des frais de } \\
\text { développement } \\
\text { (dépenses de } \\
\text { production) quand } \\
\text { forte probabilité de } \\
\text { réussite du projet }\end{array}$ & $\begin{array}{c}\text { Activation des frais de } \\
\text { développement si les } \\
\text { conditions sont réunies } \\
\text { (méthode } \\
\text { recommandée) } \\
\text { Ou } \\
\text { Inscription en charges } \\
\text { (option) }\end{array}$ & $\begin{array}{l}\text { Inscription des frais } \\
\text { de développement en } \\
\text { charges au compte de } \\
\text { résultat. } \\
\text { Interdiction de } \\
\text { l'activation. }\end{array}$ \\
\hline & se d'utilisation & $\begin{array}{l}\text { Valeur comptable i } \\
\text { (« consommations des } \\
\text { dépréciations }\end{array}$ & $\begin{array}{l}\text { thode du coût historique } \\
\text { tiale diminuée du cumul } \\
\text { vantages économiques " } \\
\text { intégration des moins-va }\end{array}$ & $\begin{array}{l}\text { es amortissements } \\
\text { uturs de l'actif) et des } \\
\text { les latentes) }\end{array}$ \\
\hline
\end{tabular}

Tableau de synthèse sur l'évaluation et la comptabilisation des brevets

Quels que soient le référentiel comptable et le mode d'acquisition, les règles de reconnaissance initiale et postérieure des brevets dans les états financiers ne peuvent que conduire à leur sousévaluation comptable au regard de leur valeur de marché. Cette situation n'a pas conduit les normalisateurs comptables à demander la publication de données supplémentaires en annexe de manière à suppléer ces inconvénients. Les informations obligatoires ne permettent donc pas de comprendre totalement la nature et le potentiel de ces actifs.

En raison des différences de traitement des incorporels selon leur mode d'acquisition, des groupes choisissent de publier de l'information financière retraitée. Leur communication financière s'appuie alors sur des mesures alternatives de performance (APM : Alternative Performance Measures) également appelées informations non-GAAP (au sens non comptables) plutôt que sur les données comptables «pures ». Par exemple, dans le secteur pharmaceutique, les dotations aux amortissements des immobilisations incorporelles sont éliminées du résultat de l'exercice de manière à ne pas avoir de différences selon que l'élément immatériel est acheté (avec une inscription à l'actif suivie d'amortissements, voire de dépréciations) ou, au contraire, développé en interne (donc non activé et non amorti pour les charges supportées avant le point de basculement). 
Pour faciliter «la compréhension de sa performance opérationnelle par la direction et les investisseurs », le groupe publie le « résultat net des activités » comme indicateur alternatif de performance. Par rapport au résultat comptable, cet APM retraite (élimine) l'amortissement et la dépréciation des actifs incorporels à durée de vie limitée comme les brevets pour faciliter «la comparaison de sa performance opérationnelle avec celle de ses pairs du secteur pharmaceutique qui inscrivent de tels actifs incorporels (principalement des brevets et des marques commerciales) à de faibles valeurs comptables (...) parce qu'ils résultent d'activités internes de recherche et développement qui ont déjà été passées en charge au cours de périodes précédentes » parce que non-activables.

Pour l'année 2018, Sanofi publie un « résultat net des activités » (information non-GAAP) de 6819 millions d'euros contre un résultat groupe (donnée comptable) de 4306 millions d'euros.

Le retraitement des amortissements et dépréciations relatif aux actifs incorporels à durée de vie limitée représente 2888 millions d'euros, soit $42 \%$ du résultat comptable.

Au regard de cette situation s'expliquant par le concept de «prudence » (conservatism) au sens large, des chercheurs ont tenté d'apprécier le caractère informationnel des données financières portant sur les immobilisations incorporelles.

\section{La revue de la littérature}

Au cours des vingt dernières années, de nombreux auteurs ont porté leur attention sur les règles de reconnaissance et d'évaluation des incorporels. À notre connaissance, il y a peu de travaux spécifiques portant sur les brevets. La problématique est néanmoins la même quelle que soit la nature de l'incorporel : brevets, logiciels, frais de développement... qui présentent un traitement comptable similaire.

Tout d'abord, des chercheurs montrent que l'absence de reconnaissance au bilan de nombreux investissements immatériels, comme les frais de recherche et développement (R\&D), se traduit par une augmentation des erreurs de prévisions de résultat des analystes financiers [GU 05] et par une asymétrie d'information entre les dirigeants et les actionnaires qui est mise à profit par les managers pour optimiser leurs décisions d'achat et de vente de titres de leur entreprise [ABO 00]. Cette situation aboutit à un coût des capitaux propres plus élevé et à une mauvaise allocation des ressources en raison d'une incitation à diminuer les investissements incorporels qui sont plutôt sousévalués par les investisseurs [LEV 05]. Cela conduit les analystes financiers à assurer un suivi plus important des firmes avec des dépenses de R\&D élevées [BAR 01]. De la sorte, ils arrivent à capter une partie de l'information relative aux immatériels non-inscrits au bilan [KIM 07]. Cela s'avère toutefois insuffisant [AMI 03].

Cette absence de reconnaissance à l'actif d'un certain nombre de dépenses immatérielles a conduit plusieurs chercheurs à s'interroger sur la pertinence de les activer (c'est-à-dire de les inscrire à l'actif). En d'autres termes, reconnaitre ces investissements au bilan comme des immobilisations incorporelles se traduirait-il par un complément d'information utile à l'investisseur? En tentant de répondre à cette question, des recherches montrent que l'activation des frais de développement des logiciels réduit l'asymétrie d'information via une réduction du bid-ask spread (la fourchette de prix des titres c'est-à-dire l'écart entre le prix demandé par le vendeur et celui offert par l'acheteur), une augmentation du taux de rotation des actions [MOH 05] et une diminution de la sous-évaluation des titres lors d'une introduction en bourse [GIV 08]. Cette situation se traduit également par des informations financières sur les incorporels plus 
représentatives des événements incorporés dans les valeurs de marché (cours et rendements boursiers), c'est-à-dire utilisés et valorisés par le marché. En d'autres termes, l'activation des frais de développement des logiciels [ABO 98] et des frais de R\&D [LEV 96] permet d'aboutir à de l'information comptable plus utile à l'investisseur.

Cette amélioration de la pertinence des données financières est d'autant plus forte que le caractère discrétionnaire des charges à activer et de la durée d'amortissement est important [CHAM 03]. Cette marge de manœuvre permet aux entreprises d'adapter au mieux le traitement comptable de leurs incorporels à leur situation propre. Finalement, en dépit du risque de comptabilité créative c'est-àdire de manipulation des comptes [DUM 15] [MAR 08], l'activation des immatériels aboutit à la publication d'une information financière plus utile en indiquant à l'investisseur les éléments immatériels sources de création de valeur future.

Dans le contexte français où les entreprises ont le choix entre une activation lorsque les conditions sont réunies et une inscription en charges au compte de résultat, l'association entre les frais de développement inscrits au bilan et les données de marché (cours et rendements boursiers) est négative [CAZ 06]. Cela signifie que les coûts de développement inscrits au bilan ne représentent pas des investissements valorisés par le marché. Ce résultat est surprenant puisque seuls les projets susceptibles de dégager des flux de revenus futurs sont activables. Cette recherche contredit les travaux précédents réalisés sur des échantillons de groupes issus de pays anglo-saxons. Dans cadre d'une comparaison internationale, ce résultat n'est pas confirmé. À l'inverse, l'allocation des frais de recherche et développement entre des charges et une reconnaissance à l'actif au bilan (lorsqu'elle est possible) semble améliorer la qualité de l'information comptable fournie par les firmes hexagonales [ZHA 02]. En comparant la value relevance des immobilisations incorporelles selon les normes comptables utilisées (françaises ou IFRS), Lenormand et Touchais (2008) démontrent, par ailleurs, que les deux référentiels comptables aboutissent à de l'information pertinente. Les nouvelles règles de reconnaissance et d'évaluation des actifs incorporels avec les IFRS semblent néanmoins apporter un complément d'information utile. En d'autres termes, elles aboutissent à des informations financières sur les incorporels plus représentatives des évènements incorporés dans les prix.

Cet apport de l'activation des éléments immatériels pour les investisseurs est également démontré pour les analystes financiers. Ainsi, Matolcsy et Wyatt (2006) observent que la capitalisation s'accompagne de plus faibles erreurs de prévisions des analystes pour les firmes présentant des incorporels importants. Anagnostopoulou (2010) et Chalmers et al. (2012) confirment cette relation positive entre l'activation des intangibles et la qualité des prévisions des analystes financiers.

Au-delà de la protection légale, déposer des brevets consiste également à envoyer un signal sur le succès de l'activité de recherche et développement et ainsi réduire l'asymétrie d'information inhérente aux projets de R\&D [LON 02]. Cela a conduit plusieurs chercheurs à tenter de confirmer le caractère informatif des données relatives aux brevets [DEN 99] [HIR 01] [PAN 11] en portant leur attention sur des mesures de quantité et de qualité : nombre de brevets déposés, nombre de citations des brevets de l'entreprise, nombre de références scientifiques indiquées dans les brevets et âge des brevets cités dans les brevets déposés. Dans un contexte où les frais de R\&D ne peuvent pas être activés (US GAAP), ces recherches montrent que les dépenses de recherche et développement sont plus pertinentes au regard des valeurs de marché (rendements et, ou cours boursiers) lorsqu'elles s'accompagnent d'innovations importantes [DEN 99] [HIR 01]. Les performances financières (flux de trésorerie et résultat) futures (et leur volatilité) semblent, par ailleurs, positivement (négativement) liées à la quantité et la qualité des brevets déposés [PAN 11]. Ces résultats soulignent le contenu informationnel important relatif aux données non financières portant sur les brevets. Ces informations permettent aux investisseurs et aux analystes financiers d'apprécier la réussite et le caractère innovant des projets de recherche. Même si l'entreprise n'a pas l'obligation 
de publier de telles données dans ses rapports financiers annuels, une divulgation volontaire devrait permettre aux lecteurs des comptes de mieux appréhender le potentiel de l'activité de R\&D en termes de flux de trésorerie futurs.

En résumé, les travaux montrent qu'une inscription au bilan des éléments immatériels réduit l'asymétrie d'information pour les investisseurs et pour les analystes financiers. Cela permet de réduire l'incertitude sur les résultats futurs en divulguant de l'information «privée » sur les projets présentant une forte probabilité de réussite qui sont alors inscrits au bilan. Cette activation signale la réussite à venir des différents projets de développement (c'est-à-dire les avantages économiques futurs) et permet de faire ressortir leur « consommation » et d'éventuelles pertes de valeur au travers des dotations aux amortissements et des dépréciations. La marge de manœuvre accordée aux groupes pour reconnaître ces incorporels au bilan semble améliorer le caractère informationnel de ces informations. En d'autres termes, rendre l'activation des frais de développement obligatoire dès lors que les conditions sont réunies, comme les règles internationales (IFRS) le prévoient, permet d'aboutir à une information financière de meilleure qualité. Ces travaux montrent également l'intérêt de communiquer sur les résultats de l'activité de recherche et son caractère innovant en divulguant notamment de l'information relative aux brevets.

\section{Conclusion}

D'une manière générale, le cadre comptable n'est pas très bien adapté pour intégrer les éléments immatériels qui constituent aujourd'hui la principale source de création de valeur des entreprises. Selon le référentiel comptable (et donc les présupposés théoriques du normalisateur comptable) et selon le mode d'acquisition (achat versus fabrication en interne), les modalités de reconnaissance et d'évaluation au bilan s'avèrent très différentes. Les brevets, au même titre que les autres investissements immatériels, sont concernés par ces difficultés.

L'analyse des règles comptables relatives au brevet fait ressortir les deux principaux enseignements suivants :

-Les brevets sont toujours sous-évalués au niveau du bilan en raison de l'interdiction de réévaluer à la hausse leur valeur comptable en présence de plus-values latentes. Cette sousévaluation est plus importante dans le cas de brevets ayant fait l'objet d'un développement interne. Les dépenses de production de l'innovation ne peuvent pas être activées avec les US GAAP et seulement partiellement avec les IFRS puisque cela suppose au préalable de lever l'incertitude sur la réussite du projet.

- Les règles comptables se traduisent également par des impacts sur le résultat publié. L'absence de reconnaissance des frais de développement du brevet au bilan s'accompagne d'une constatation immédiate en charges alors qu'il y a un étalement par le biais des amortissements et des éventuelles pertes de valeur dans le cas contraire. Cela signifie qu'il y a un décalage dans le temps entre l'enregistrement des coûts de développement en charges et des recettes générées par le projet. Cela ne permet pas de rattacher à chaque exercice le supplément de marges lié aux innovations.

Les travaux de recherche montrent que l'activation des frais de développement apporte un complément d'information utile à l'investisseur en divulguant de l'information " privée » sur les projets présentant une forte probabilité de réussite. Même si cette activation présente un caractère discrétionnaire, elle permet d'avoir une plus grande visibilité sur les flux de trésorerie futurs susceptibles d'être générés par les projets en cours. Réduire l'asymétrie d'information suppose également de communiquer sur les résultats obtenus par l'activité de R\&D en diffusant notamment de l'information qualitative et quantitative relative aux brevets. 


\section{Bibliographie}

[ABO 00] ABOODY D., LEV B., «Information asymmetry, R\&D, and insider gains », The Journal of Finance, vol. LV, $\mathrm{n}^{\circ} 6$, p. 2747-2766, 2000.

[ABO 98] ABOODY D., LEV B., « The value relevance of intangibles: The case of software capitalization », Journal of Accounting Research, vol. 36 (suppl.), p. 161-191, 1998.

[AMI 03] AmiR E., Lev B., Sougiannis T., « Do financial analysts get intangibles? », European Accounting Review, vol. 12, n 4, p. 635-659, 2003.

[ANA 10] ANAGnostopoulou S.C., "Does the capitalization of development costs improve analyst forecast accuracy? Evidence from the UK », Journal of International Financial Management \& Accounting, vol. 21, $\mathrm{n}^{\circ} 1, \mathrm{p}$. $62-83,2010$.

[BAR 01] BARTH M.E., KASNIK R., MC NiCHOLS M.F., « Analyst coverage and intangible assets », Journal of Accounting Research, vol. 39, n 1, p. 1-34, 2001.

[CAZ 06] CAZAVAN-JENY A., JEANJEAN T., «The negative impact of R\&D capitalization: A value relevance approach », European Accounting Review, vol. 15, n 1, p. 37-61, 2006.

[CHA 12] CHALMER K., ClinCH G., GODFREY J.M., WEI Z., « Intangible assets, IFRS and analysts’ earnings forecasts », Accounting and Finance, vol. 52, p. 691-721, 2012.

[CHA 03] ChAmbers D., Jennings R., THOMPSON R., « Managerial discretion and accounting for research and development costs », Journal of Accounting, Auditing and Finance, vol. 18, n 1, p. 79-113, 2003.

[DEN 99] Deng Z., Lev B., NARIN F., " Science and technology as predictors of stock performance », Financial Analysts Journal, vol. 55, n 3, p. 20-32, 1999.

[DUM 15] Dumas G., MARTINEZ I., «IAS 38 et activation des dépenses de développement Comptabilisation opportuniste ou informative ? », Revue Française de Gestion, n² 249, p. 93-111, 2015.

[GIV 08] Givoly D., SHI C., « Accounting for software development costs and the cost of capital: Evidence from IPO underpricing in the software industry », Journal of Accounting, Auditing and Finance, vol. 23, n 2, p. 271-303, 2008.

[GU 05] GU F., WANG W., « Intangible assets, information complexity, and analysts' earnings forecasts, Journal of Business Finance and Accounting », vol. 32, n 9-10, p. 1673-1702, 2005.

[HIR 01] HIRSCHEY M., RICHARDSON V.J., SCHOLZ S., «Value relevance of nonfinancial information: The case of patent data », Review of Quantitative Finance and Accounting, vol. 17, p. 223-235, 2001.

[IAS 16] IASB, IFRS 16 - Contrats de location, Londres, 2016.

[IAS 04] IASB, IAS 38 - Immobilisations incorporelles, Londres, 2004.

[KIM 07] KIMBROUGH M.D., « The influences of financial statement recognition and analyst coverage on the market's valuation of R\&D capital », The Accounting Review, 2007, vol. 82, n 5, p. 1195-1225, 2007.

[LEN 08] LENORMAND G., TOUCHAIS L., «La pertinence des actifs incorporels avec les IFRS », Finance Contrôle Stratégie, vol. 11, n² 2, p. 173-201, 2008.

[LEV 05] LeV B., Bharat S., Sougiannis T., « R\&D reporting biases and their consequences », Contemporary Accounting Research, vol. 22, n 4, p. 977-1026, 2005.

[LEV 96] LEV B., Sougiannis T., « The capitalization, amortization and value relevance of R\&D », Journal of Accounting and Economics, vol. 21, p. 107-138, 1996.

[LEV 03] LEV B., ZAMBON S., «Intangibles and intellectual capital: An introduction to a special issue », European Accounting Review, vol. 12, n 4, p. 597-603, 2003.

[LEV 99] LEV B., ZAROWIN P., «The boundaries of financial reporting and how to extend them », Journal of Accounting Research, vol. 37, n² 2, p. 353-385, 1999.

[LON 02] LONG C., «Patent signals », University of Chicago Law Review, vol. 69, n² 2, p. 625-679, 2002.

[MAT 06] MATOLCSY Z., WyATt A. « Capitalized intangibles and financial analysts », Accounting and Finance, vol. 46, p. 457-479, 2006.

[MAR 08] Markarian G., Pozza L., Prencipe A., « Capitalization of R\&D costs and earnings management: Evidence from Italian companies », The International Journal of Accounting, vol. 43, n 3, p. 246-267, 2008. 
[MOH 05] MOHD E., « Accounting for software development costs and information asymmetry », The Accounting Review, vol. 80, n 4, p. 1211-1231, 2005.

[PAN 11] PANDIT S., WASLEY C.E., ZACHT T., « The effect of research and development (R\&D) inputs and outputs on the relation between the uncertainty of future operating performance and R\&D Expenditures », Journal of Accounting, Auditing \& Finance, vol. 26, p. 121-144, 2011.

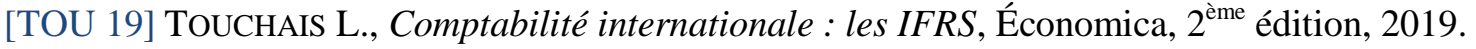

[ZHA 01] ZHAO R., «Relative value relevance of R\&D reporting: An international comparison », Journal of International Financial Management and Accounting, vol. 13, n 2, p. 153-174, 2002. 